Rapport - Société canadienne d'histoire de l'Église catholique

\title{
Un philanthrope d'autrefois : Antoine-Olivier Berthelet
}

\section{Léon Trépanier}

Volume 28, 1961

URI : https://id.erudit.org/iderudit/1007374ar

DOI : https://doi.org/10.7202/1007374ar

Aller au sommaire du numéro

Éditeur(s)

La Société canadienne d'histoire de l'Église catholique

ISSN

0318-6148 (imprimé)

1927-7075 (numérique)

Découvrir la revue

Citer cet article

Trépanier, L. (1961). Un philanthrope d'autrefois : Antoine-Olivier Berthelet. Rapport - Société canadienne d'histoire de l'Église catholique, 28, 19-25.

https://doi.org/10.7202/1007374ar

Tous droits réservés @ La Société canadienne d'histoire de l'Église catholique, 1963
Ce document est protégé par la loi sur le droit d'auteur. L'utilisation des services d'Érudit (y compris la reproduction) est assujettie à sa politique d'utilisation que vous pouvez consulter en ligne.

https://apropos.erudit.org/fr/usagers/politique-dutilisation/ 


\section{Un philanthrope d'autrefois: Antoine-Olivier Berthelet}

Dans le cadre de ce congrès, il n'est pas hors de propos de faire revivre le nom d'un montréalais qui fut une providence pour un grand nombre de nos institutions naissantes.

A une époque où le rôle de philanthrope semble dévolu de plus en plus à l'Etat, on a peine à croire qu'autrefois, chez les nôtres, des fortunes complètes étaient distribuées au gré des besoins d'institutions religieuses ou de bien-être social.

Ce fut pourtant le cas d'Antoine-Olivier Berthelet, dont les libéralités ont rendu le nom presque légendaire dans le domaine de la philanthropie.

L'auteur des Grandes Familles au Canada, le classe au rang « des bienfaiteurs de ce continent ".

«Pour l'époque », a écrit E.-Z. Massicotte, "ce philanthrope fut d'une munificence qui égale presque celle de certains millionnaires écossais et américains."

A l'occasion de son $72^{\circ}$ anniversaire de naissance, monsieur Berthelet recevait des mains de Mgr Cyprien Tanguay, auteur du " Dictionnaire Généalogique des Familles Canadiennes" l'Arbre de sa famille, accompagné d'une pièce de vers dont on trouve le texte au-dessous du nom de l'ancêtre François Berthelet.

La famille Berthelet, originaire de la ville de Genève, Suisse, avait une grande vénération pour saint François de Sales, et c'est pourquoi, selon Mgr Tanguay, le nom de François se rencontre plusieurs fois parmi ses ascendants.

Antoine-Olivier Berthelet était, par atavisme, porté à dispenser ses biens au profit de ceux à qui la maladie ou la pauvreté avaient réservé un mauvais sort.

Le premier testament de Pierre Berthelet, père de notre philanthrope, en date du 22 octobre 1806, nous révèle l'âme généreuse du négociant montréalais. Aux pauvres et aux enfants trouvés de l'Hôpital Général des Sœurs Grises, aux pauvres de la paroisse et aux pauvres de l'Hôtel-Dieu, il est attribué des secours substantiels, même si dans les trois codicilles qui s'échelonnent jusqu'en 1826, le testateur s'occupe surtout du sort de sa descendance.

Pierre Berthelet, natif de Montréal, s'était amassé une petite fortune dans le commerce de fourrures au Michigan et, à Détroit, il avait épousé le 24 juillet 1775, Marie-Françoise Meloche, de Lachine, qui 
mourut moins d'un an plus tard, laissant un enfant, Henri qui vécut toute sa vie aux Etats-Unis. Etant retourné à Montréal, Pierre Berthelet convola une seconde fois, 2 février 1779, à Boucherville, avec demoiselle Marguerite Viger. C'est de cette seconde union que naquit Antoine-Olivier, dont le baptême fut célébré à Notre-Dame le 25 mai 1798.

Ici une parenthèse pour signaler que dans nombre de documents le nom de son père est suivi du surnom \& dit Savoyard ". Mon ancien

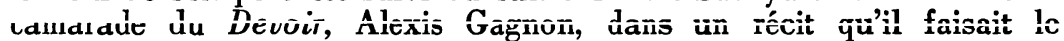
10 mars 1928, du désastreux incendie de 1852 à Montréal, rappelait les dons considérables qu'Antoine-Olivier Berthelet avait fait aux institutions et, parlant de son père, il nous dit pourquoi on l'appelait Pierre Berthelet dit le Savoyard.

" On l'avait surnommé ainsi, dit-il, parce qu'il s'était fait une spécialité de louer des poêles lorsque les froids de l'automne étaient arrivés. " "Berthelet le Savoyard ouvrait alors son entrepôt de poêles et de feuilles à tuyaux et, pendant deux mois, les poêles à deux ponts se baladaient dans les rues de Montréal. Les locataires payaient une menue redevance et, au printemps, rapportaient le poêle. *

Cette note de Gagnon m'a permis de connaître l'origine de ce terme : savoyard. Le « Dictionnaire de l'Académie » nous apprend, en effet, que la Savoie fournissant autrefois un grand nombre d'hommes exerçant le métier de ramoneurs, par extension, on appelle " savoyard ", un ramoneur.

L'un des frères d'Antoine-Olivier fut médecin et mourut relativement jeune en 1847 : son frère consanguin, Henri, de Détroit, eut une carrière si agitée que tantôt on le voit figurer au livre des Patriotes de 1837 d'Aegidius Fauteux, bien que ce dernier avoue n'avoir rien découvert de bien précis à ce sujet, ou bien encore, par l'un de ses descendants, dans une petite ville du Dakota Nord.

Il faut tout de même consacrer quelques lignes à Thérèse, sœur unique d'Antoine-Olivier, qui, pendant douze ans, vécut une vie de recluse, dans une petite chambre de l'Asile de la Providence après avoir remis toute sa fortune entre les mains de son frère afin de permettre à ce dernier de continuer ses libéralités envers nos institutions.

Elle avait 71 ans lorsqu'elle prit cette décision et révéla à son frère qu'elle désirait vivre désormais, solitaire, pauvre et oubliée. C'est ainsi qu'elle vévut, en effet, durant les douze dernières années de sa vie après avoir fait don d'une somme de $\$ 19,400$. aux Religieuses comme faible compensation de leur hospitalité.

Le père de notre philanthrope décéda en 1830 et il y a lieu de croire qu'Antoine-Olivier ne tarda pas à faire fructifier l'héritage qu'il avait reçu, puisque cinq ans après commençait la série de ses libéralités qui devaient se continuer jusqu'à son décès.

Mais voyons qu'elle fut la carrière de cet homme de bien. 
A l'âge de 8 ans, Antoine-Olivier entre au collège de Montréal et, après quelques années de cours classique, se voit possesseur d'une fortune qu'il a le devoir de faire fructifier et d'administrer sagement.

Le 30 octobre 1822, il épouse en premières noces Marie-Angéline Amélia, fille de Louis Chaboillez, notaire. Le 23 janvier 1823, était baptisée son unique enfant, Marie-Amélie qui épousa, en 1841, FrançoisAlfred-Chartier Larocque. Madame Larocque mourut à Paris en 1856.

Son second mariage avec Charlotte, fille de l'honorable Louis Guy, en 1855 , ne lui ayant laissé aucune postérité, il lui fut facile de disposer de ses biens comme il l'entendait.

La vie politique attira quelque peu Olivier Berthelet et, sur les instances de ses amis, il se présenta dans Montréal-Est, en avril 1832, et fut élu député à l'Assemblée législative. La situation politique à ce moment était quelque peu troublée, et il semble bien que l'ambiance parlementaire ne s'accommoda guère à son tempérament d'homme d'affaires. Deux ans plus tard, ayant perdu sa mère, il abandonnait son mandat politique, ce qui ne l'empêcha pas, cependant, de s'enrôler dans la fameuse Association des Fils de la Liberté. Mais lorsqu'éclata la Rébellion, Olivier Berthelet venait de se retirer des affaires et avait déjà commencé à distribuer sa fortune au soutien des bonnes œuvres.

Deux ans auparavant, il avait fait don à madame Gamelin, fondatrice de la Providence, d'une maison sise rue Sainte-Catherine, près de l'ancien évêché qui était, comme on le sait, attenant à la cathédrale, coin Sainte-Catherine et Saint-Denis. Ce don permit à madame Gamelin d'y loger plus d'une vingtaine d'infirmes, dès le $1^{\text {or }}$ mai 1835.

Olivier Berthelet ne prit aucune part au soulèvement de 1837. Il était d'ailleurs, à ce moment, l'un des commissaires chargés de l'administration du canal Lachine, fonction qu'il occupa jusqu'après l'amnistie. Lorsque la paix revint en 1840, que l'administration de la ville par des magistrats prit fin et que Montréal obtint sa deuxième charte, Olivier Berthelet fut élu membre du conseil et fit partie du Comité des chemins et du Comité des marchés. Le 3 juin 1842, il était élu échevin en remplacement de l'échevin Quesnel, décédé. Ce fut la fin de son stage dans le domaine municipal.

La politique intéressait peu Olivier Berthelet, avons-nous dit. On s'en rend compte par la lecture des procès-verbaux du conseil municipal de Montréal en 1841 et 1842 et les journaux de la Chambres d'Assemblée durant les années 1832-1834. Berthelet ne prit aucune part active aux discussions, soit au conseil municipal ou au parlement. Sa nomination au Conseil législatif par Lord Sydenham, le 9 juin 1841, devait être le point final de sa carrière d'homme public car quinze jours plus tard, Berthelet démissionnait et annonçait son retrait de la vie politique. Voici d'ailleurs la lettre que $M$. Berthelet fit tenir au gouverneur général à la date du 26 juin 1841 .

- I have the honor of informing Your Excellency that for the reason which on a former occasion I had the honor of stating to Your Excellency, it is not in my power to accept at present a seat in the 
Legislative Council of this Province, and that I formally resign the seat therein to which it has been Your Excellency pleasure to call me.» Il faut dire ici, fait remarquer M. Pierre-Georges Roy ${ }^{1}$ qu'en offrant un siège au Conseil législatif à $M$. Berthelet, le gouverneur Sydenham avait posé une condition, celle, pour le titulaire d'assister régulièrement aux séances du conseil et de ne pas considérer cette charge comme simplement honorifique.

- Le cas de M. Berthelet n'est peut-être pas unique, conclut M. Roy, mais ee n'est pas souvent qu'on voit un citoyen refuser un siège au Conseil législatif. »

Quelques jours plus tard, monsieur Berthelet mariait sa fille unique à François-Alfred-Chartier Larocque.

Désormais, Olivier Berthelet n'aura plus que deux préoccupations : l'administration de ses biens et la distribution de son immense fortune à des institutions.

Il a, à ce moment, un bureau d'affaires rue Saint-Jacques et habite rue Notre-Dame passé McGill.

Depuis longtemps il s'était débarrassé d'une terre qu'il possédait dans la seigneurie de Boucherville et songeait à disposer peu à peu de ses propriétés en ville.

Les Sœurs de la Providence occupaient l'attention de monsieur Berthelet, surtout depuis que la législature provinciale avait accordé à l'institut l'incorporation civile et que Thérèse, sa sœur, s'était jointe à madame Gamelin, et dix autres femmes d'élite pour former la Corporation de l'Asile des femmes âgées et infirmes de Montréal.

Les efforts entrepris par les Jésuites pour fonder un noviciat au Sault-au-Récollet ne laissèrent pas monsieur Berthelet indifférent. Dans son " Histoire de la paroisse du Sault-au-Récollet ", l'abbé Charles-P. Beaubien parlant de cette fondation écrit que "M. Berthelet fut l'instrument que saint Joseph choisit pour réaliser cette fondation 》.

En août 1842, les pères Oblats sont transférés à Longueuil dans une maison donnée par Olivier Berthelet, en mémoire de son parent, l'abbé Augustin Chaboillez, ancien curé de Longueuil. La maison en question avait appartenu au curé.

La Presse du 13 novembre 1931, annonçant la démolition d'un pâté de maisons dans le quadrilatère formé par les rues Saint-Jacques, Windsor, Saint-Antoine et Cathédrale, déplorait la disparition de l'hospice Saint-Joseph, rue de la Cathédrale et de la chapelle dédiée à saint Joseph, qui se dressait tout à côté de l'hospice, petite chapelle où le bon Frère André était allé souvent s'agenouiller.

Ces deux immeubles avaient pu s'édifier grâce à la générosité de la famille Berthelet.

1 L'Hôpital Général de Montréal « Sceurs Grises», par Sour Clémentine Drouin, s.g.m., 1943. Tome I, pp. 18-21. 
A l'automne de 1841, M. Olivier Berthelet avait fait construire, angle des rues Saint-Jacques et de la Cathédrale, une vaste maison en bois destinée à recevoir les pauvres, particulièrement les veuves sans ressources et les femmes incapables de payer loyer. En 1846, Mgr Prince célébrait la première messe dans la petite chapelle attenante et vers la même époque, M. Gottefrey, premier chapelain, fondait une association de dames de la charité dont Mlle Berthelet, sœur du fondateur, fut la première présidente.

A ce sujet, nous lisons dans l'Histoire des Sœurs Grises de Montréal, ${ }^{1}$ ce qui suit : «M. Pierre-Louis Billaudèle, supérieur du Séminaire, demanda un jour à M. Berthelet s'il n'agréerait pas qu'on remplaçat les demoiselles directrices par des sours \&Grises »: l'heure de la Providence avait sonné.

- Le 23 décembre 1853, le Fondateur (M. Berthelet) alla lui-même offrir son instilution à notre Mère Julie Deschamps. Il avait même dressé l'acte de donation que sa fille, madame Larocque, qui l'accompagnait, déposa sur le tombeau de notre Vénérable Mère d'Youville... Le 9 mars 1854, Mère Julie Deschamps écrivait à Mère Valade, supérieure de la maison de Saint-Boniface :

- M. Berthelet vint nous offrir, le 23 décembre, anniversaire de la mort de notre Vénérable Mère d'Youville, son Hospice Saint-Joseph, avec deux autres maisons de bois." Ailleurs on voit qu'en 1856, monsieur Berthelet dotait l'Hospice d'une propriété évaluée à $\$ 3,260$. Côte Saint-Antoine. Pour ces nombreuses libéralités, monsieur Berthelet devait obtenir l'honneur insigne de Commandeur de Sa Sainteté Pie IX.

Grâce à la libéralité de monsieur Berthelet et de sa sœur Thérèse, on construisait, tout à côté de l'hospice, en 1862, l'église Saint-Joseph qui fut consacrée par Mgr Bourget, le 15 octobre 1863.

C'est dans ce pieux sanctuaire qu'était érigée canoniquement le 18 août 1867, la Confrérie Saint-Joseph, affiliée aussitôt après à l'archiconfrérie de Beauvais, France. Quelques années plus tard, l'hospice fut transformé en école d'enseignement ménager.

Mais peut-on oublier que M. Olivier Berthelet fut aussi l'un des grands bienfaiteurs de l'Institut des Sœurs de Miséricorde de Montréal, qu'il en fut le " pourvoyeur de la première heure et de toujours ».

Dans son histoire de la communauté, l'abbé Elie-J. Auclair résume l'œuvre du philanthrope envers cette fondation plus que séculaire et il puise, dans les annales de l'institut, ces quelques lignes, inspirées par le sentiment intense de reconnaissance des religieuses : "Ami de toutes les bonnes œuvres, M. Berthelet savait leur faire une large part des richesses que le ciel lui avait départies... La communauté lui est redevable de vingt mille piastres qu'il nous a données tant pour nos bâtisses que pour nos différentes réparations, sans compter bien des petits dons et une quantité de meubles... Il n'y a personne dans toute la cité à qui cet homme n'ait fait du bien. Ceux qui, ayant le pain matériel en abondance, n'avaient pas besoin de ses aumônes, recevaient de sa vie de citoyen et de chrétien exemplaire, les lumières qui édifient 
et rendent meilleurs... Les services qu'il nous a rendus pendant plus de vingt-cinq ans, sont de ceux dont Dieu seul peut être le juste rémunérateur. »

Bien que les libéralités du philanthrope semblent se confiner à Montréal, elles dépasseront souvent les frontières de la province et on a rappelé que les missions de la Baie d'Hudson eurent un insigne protecteur dans la personne de Olivier Berthelet. En 1850, un an après qu'il eût fait les frais d'incorporation des Frères de Saint-Laurent, il

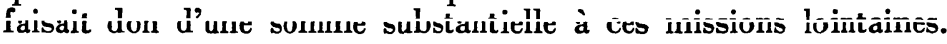

En 1852, il achète du shérif, pour Mgr l'évêque de Montréal, au prix de $\$ 16,000$. le collège baptiste, rue Guy, pour y établir l'hôpital Saint-Patrice. On sait que cet édifice fit partie plus tard du pensionnat du Mont Sainte-Marie. " Il est possible, lit-on dans l' "Annuaire de Ville-Marie ", que M. Berthelet ait largement contribué à cet achat."

En 1861, monsieur Berthelet fait don d'un terrain et d'une maison pour la fondation de l'hospice Saint-Antoine rue Labelle, près de Dorchester. La construction de l'aile nord-est du monastère du Bon Pasteur, au coût de $\$ 15,200$. en 1861 fut réalisé grâce au philanthrope qui en avait assumé le coût. Le 22 octobre 1863, il fait don aux pères Jésuites, rue Bleury, d'une propriété d'un arpent et demi d'une valeur de plus de $\$ 20,000$. pour y asseoir une église suivant la forme et les proportions du Gésu de Rome, d'après ce qu'on lit dans les journaux du temps.

En 1868, ayant pris une part active à l'organisation des Zouaves Pontificaux, il envoie à ses propres frais vingt volontaires en Italie. L'année précédente, son petit-fils, Alfred Larocque, membre de cette milice papale, avait été blessé à la bataille de Mentana.

Les Frères de la Charité furent probablement ceux qui furent particulièrement l'objet des libéralités du philanthrope puisqu'on lit qu'il souscrivit plus de $\$ 120,000$. pour la fondation de leur Institut, rue Demontigny, aujourd'hui l'Institut Saint-Antoine, rue Sherbrooke est.

A la liste de ces dons, il faut en ajouter plusieurs autres que l'on retrace ici et là au cours des recherches. Tantôt, c'est un don de $\$ 1,000$. à Mgr Taché ou une somme assez substantielle pour maison de retraite près de Pittsburg, ou bien encore une somme de $\$ 2,500$. aux orphelins de Saint-Joseph ou une cloche à l'Hôtel-Dieu.

Faire le bilan des générosités de monsieur Berthelet envers nos institutions a été avéré chose impossible pour tous ceux qui ont voulu s'imposer cette tâche, mais s'il est vrai, comme on l'a affirmé, qu'Antoine-Olivier Berthelet a dispensé, de son vivant, près d'un million de dollars à des ouvres de charité, d'assistance sociale et d'enseignement, il faut donner la palme au philanthrope montréalais, à sa femme et à sa søur Thérèse.

Notre concitoyen ne put échapper aux charges honorifiques, sorte de tribut que doit souvent payer celui qui se dévoue opiniâtrement pour le bien des siens. 
Antoine-Olivier Berthelet fut président général de la Société SaintJean-Baptiste en 1863. A l'Union Catholique, lors de l'organisation d'un bataillon de Zouaves Pontificaux, on lui confia des initiatives qu'il s'efforça de réaliser le mieux possible. Il fut même lieutenant-colonel de milice vers 1848, mais ce ne fut pas pour longtemps. Son tempérament pacifique, sa nature conciliante, et fort probablement l'état de malaise qui existait à ce moment à Montréal, un an avant l'incendie du Parlement par un groupe de tories courroucés, une situation qui l'aurait forcé de sortir son épée du fourreau, écourtèrent sa carrière de milicien. Aussi fut-il colonel de passage comme il avait été conseiller législatif pendant quelques jours. Quand il s'éteignit, le 25 septembre 1872, à l'âge de 74, ans, dans la superbe maison qu'il occupait, 291, rue Dorchester, coin de la petite rue Labelle, il était doyen du corps des marguillers de Notre-Dame.

Le Nouveau-Monde lui consacra plusieurs colonnes, résumant ainsi sa carrière : "Ses richesses, sa position, lui permettaient de vivre avec un certain éclat. Il se refusa tout cependant, au bénéfice des bonnes œuvres. La frugalité de sa table, la simplicité de sa tenue, la modestie qui régnait partout dans sa maison, n'avaient point d'autre but que celui de pouvoir secourir ses semblables. »

C'était bien là le témoignage qui lui était dû et qui résume un peu ce qu'il avait été. Je me rappelle avoir lu dans l' a Annuaire de VilleMarie» l'incident qui avait porté Olivier Berthelet à ouvrir un jour largement sa bourse, aux bonnes Sœurs de la Providence. On l'avait invité à faire une visite à l'établissement où les religieuses hospitalisaient des infirmes. L'une d'entre elles s'avança tout à coup vers le visiteur et lui dit : « Monsieur, vous qui avez des maisons, si vous voulez, vous nous en donnerez une. " Berthelet se sentit ému et donna à madame Gamelin la spacieuse maison dont il était propriétaire rue Sainte-Catherine, près de Berri.

Les obsèques de l'honorable Olivier Berthelet furent célébrées en l'église Notre-Dame, le 28 septembre 1872. La communauté des Sœurs Grises était représentée par quarante religieuses et des groupes d'orphelins et d'orphelines. Les autres communautés religieuses y étaient aussi largement représentées. Sa Grandeur Mgr Bourget et un nombreux clergé remplissaient le chœur.

Après le chant du Libera, Mgr Bourget récita les prières de l'Eglise sur la fosse du défunt creusée au-dessous de la chapelle Saint-Joseph de la rue Cathédrale. Lors de l'expropriation de cette église, ses restes furent transportés à la crypte de la maison mère des Sœurs Grises, rue Guy.

Telle est, rapidement esquissée, la carrière d'un montréalais dont l'humaine compréhension de la plus belle des vertus devait faciliter la floraison de nos grandes institutions de bien-être social.

Léon TRÉPANIER 